Marquette University

e-Publications@Marquette

3-2005

\title{
Both Nucleophile and Substrate Bind to the Catalytic Fe(II)-Center in the Type-II Methionyl Aminopeptidase from Pyrococcus
} furiosus

\author{
Alicja J. Copik \\ Utah State University \\ Sarah Waterson \\ Utah State University \\ Sabina I. Swierczek \\ Utah State University \\ Brian Bennett \\ Marquette University, brian.bennett@marquette.edu \\ Richard C. Holz \\ Marquette University, richard.holz@marquette.edu
}

Follow this and additional works at: https://epublications.marquette.edu/physics_fac

Part of the Physics Commons

\section{Recommended Citation}

Copik, Alicja J.; Waterson, Sarah; Swierczek, Sabina I.; Bennett, Brian; and Holz, Richard C., "Both Nucleophile and Substrate Bind to the Catalytic Fe(II)-Center in the Type-II Methionyl Aminopeptidase from Pyrococcus furiosus" (2005). Physics Faculty Research and Publications. 31.

https://epublications.marquette.edu/physics_fac/31 
Marquette University

e-Publications@Marquette

\title{
Physics Faculty Research and Publications/College of Arts and Sciences
}

This paper is NOT THE PUBLISHED VERSION; but the author's final, peer-reviewed manuscript. The published version may be accessed by following the link in the citation below.

Inorganic Chemistry, Vol. 44, No. 5 (1 March 2005): 1160-1162. DOI. This article is @ American Chemical Society Publications and permission has been granted for this version to appear in $\underline{\mathrm{e}}-$ Publications@Marquette. American Chemical Society Publications does not grant permission for this article to be further copied/distributed or hosted elsewhere without the express permission from American Chemical Society Publications.

\section{Both Nucleophile and Substrate Bind to the Catalytic Fe(II)-Center in the Type-II Methionyl Aminopeptidase from Pyrococcus furiosus}

\author{
Alicja J. Copik \\ Department of Chemistry and Biochemistry, Utah State University, Logan, Utah \\ Sarah Waterson \\ Department of Chemistry and Biochemistry, Utah State University, Logan, Utah \\ Sabina I. Swierczek \\ Department of Chemistry and Biochemistry, Utah State University, Logan, Utah \\ Brian Bennett \\ Department of Biophysics, Medical College of Wisconsin, Milwaukee, Wisconsin \\ Richard C. Holz \\ Department of Chemistry and Biochemistry, Utah State University, Logan, Utah
}




\section{SUBJECTS:}

Adducts, Metals, Substitution reactions, Ions

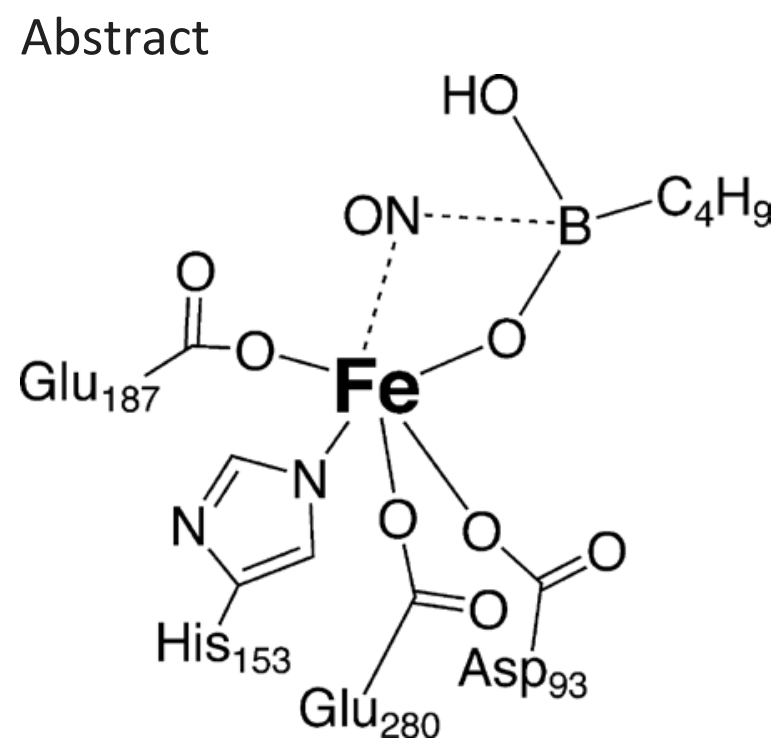

Metalloproteases utilize their active site divalent metal ions to generate a nucleophilic water/hydroxide. For methionine aminopeptidases (MetAPs), the exact location of this nucleophile, as well as of the substrate, with respect to the active site metal ion is unknown. In order to address this issue, we have examined the catalytically competent Fe(II)-loaded form of PfMetAP-II ([Fe(PfMetAP-II)]) in the absence and presence of both nitric oxide (NO) and the substrate-analogue inhibitor butaneboronic acid (BuBA) by kinetic and spectroscopic (EPR, UV-vis) methods. NO binds to [Fe(PfMetAP-II)] with a $K_{\mathrm{d}}$ of $200 \mu \mathrm{M}$ forming an $\{\text { FeNO }\}^{7}$ complex. UV-vis spectra of the resulting [Fe(PfMetAP-II)]-NO complex indicate that the Fe(II) ion is six coordinate. These data suggest that NO binding occurs without displacing the bound aquo/hydroxo moiety in [Fe(PfMetAP-II)]. On the basis of EPR spectra, the resulting Fe-NO complex is best described as $\mathrm{NO}^{-}(S=1)$ antiferromagnetically coupled to a highspin Fe(III) ion $(S=5 / 2)$. The addition of BuBA to [Fe(PfMetAP-II)]-NO displaces the coordinated water molecule forming a six-coordinate adduct. EPR data also indicate that an interaction between the bound NO and BuBA occurs forming a complex that mimics an intermediate step between the Michaelis complex and the tetrahedral transition-state.

\section{Synopsis}

The Fe(II)-loaded methionine aminopeptidase from Pyrococcus furiosus (PfMetAP-II) was examined in the absence and presence of both nitric oxide (NO) and the substrate-analogue inhibitor butaneboronic acid (BuBA) by kinetic and spectroscopic (EPR, UV-vis) methods. The addition of BuBA to [Fe(PfMetAP-II)]-NO displaces the coordinated water; however, BuBA likely undergoes nucleophilic attack by NO', mimicking an intermediate step between the Michaelis complex and the tetrahedral transition-state.

Methionyl aminopeptidases (MetAPs) represent a unique class of protease that is capable of removing Nterminal methionine residues from nascent polypeptide chains. ${ }^{1-3}$ The importance of understanding the catalytic mechanism of MetAPs is underscored by the recent observation that MetAPs are the target for anticancer drugs that inhibit angiogenesis, one of which is in third stage clinical trials. ${ }^{4}$ Thus, the design of mechanism-based inhibitors is critically important in the development of drugs that prevent tumor vasculature formation, growth, and proliferation. A common mechanistic feature for metalloproteases is a metal bound water molecule that can be ionized to an hydroxide, which then functions as the nucleophile during catalytic turnover. In order to 
determine if a metal-centered water/hydroxide exists in MetAPs, we have examined the catalytically competent Fe(II)-loaded form of the MetAP from Pyrococcus furiosus (PfMetAP-II) in the absence and presence of nitric oxide (NO) and the substrate-analogue inhibitor butaneboronic acid (BuBA).

$\mathrm{NO}$ is known to reversibly bind to high-spin Fe(II) ions within enzyme active sites and to alter their electronic properties allowing spectroscopic characterization of an otherwise spectroscopically silent $S=2$ metal center. NO binds to the five-coordinate Fe(II) center ${ }^{5}$ in the resting form of [Fe(II)(PfMetAP-II) $]^{6}$ with a $K_{d}$ value of 200 $\mu \mathrm{M}$, and the resulting [Fe(PfMetAP-II)]-NO complex is inactive. The direct interaction of NO with the Fe(II) center in [Fe(PfMetAP-II)] was confirmed by electronic absorption spectroscopy (Figure 1). The electronic absorption spectrum of the [Fe(PfMetAP-II)]-NO complex exhibits three bands at $\sim 600 \mathrm{~nm}\left(110 \mathrm{M}^{-1} \mathrm{~cm}^{-1}\right), 430$ $\mathrm{nm}\left(595 \mathrm{M}^{-1} \mathrm{~cm}^{-1}\right)$, and $\sim 350 \mathrm{~nm}\left(1200 \mathrm{M}^{-1} \mathrm{~cm}^{-1}\right)$. The positions and molar absorptivities of these bands are typical of electronic dipole, spin-allowed NO $\pi^{*} \rightarrow \mathrm{Fe}^{3+}$ LMCT transitions for a six-coordinate Fe(II)-NO center. ${ }^{7-}$ ${ }^{9}$ The electronic properties of [Fe(PfMetAP-II)]-NO were further investigated by EPR spectroscopy (Figure 2). [Fe(PfMetAP-II)]-NO exhibits an EPR signal with $g_{\text {eff }}$ values of 4.05, 3.89, and 2.0, typical of Fe(II)-NO adducts of the $\{\text { FeNO }\}^{7}$ type.${ }^{10}$ The spectrum of [Fe(PfMetAP-II)]-NO was simulated assuming $S=3 / 2, M_{S}=| \pm 1 / 2\rangle$, an isotropic $g=2.014, E / D=0.0145$, and $D \gg B g H S$ (an arbitrary value of $50 \mathrm{~cm}^{-1}$ was used). The nonzero $E / D$, evident in the spectrum as the splitting of the $g_{x}$ and $g_{y}$ features, is typical for protein centered $\left\{\mathrm{FeNO}^{7}\right.$ adducts (generally 0.015-0.006). These data confirm that NO directly interacts with the catalytic Fe(II) ion in the active site of $[\mathrm{Fe}($ PfMetAP-II)].

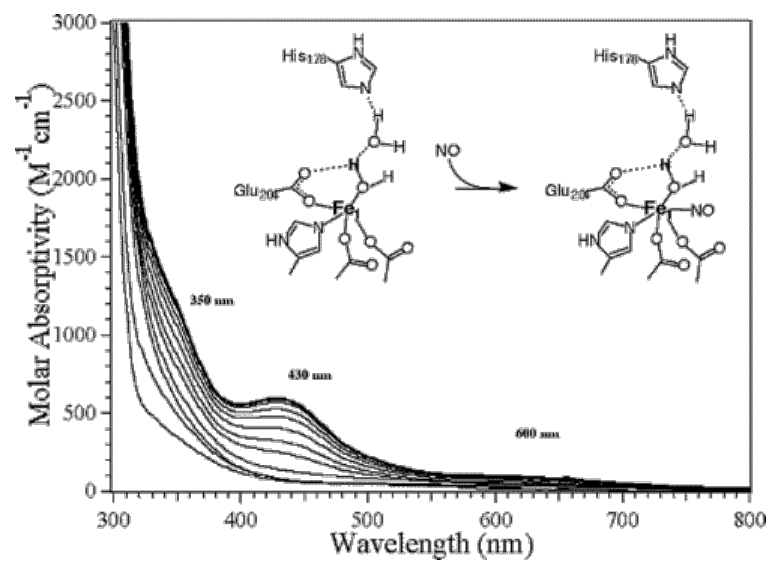

Figure 1 Electronic absorption spectrum of [Fe(PfMetAP-II)]-NO recorded at 1.5 and $5 \mathrm{~min}$ and then in $10 \mathrm{~min}$ intervals up to $95 \mathrm{~min}$. Inset: Proposed NO binding to [Fe(PfMetAP-II)]. NO was added anerobically as the Spermine NONOate.

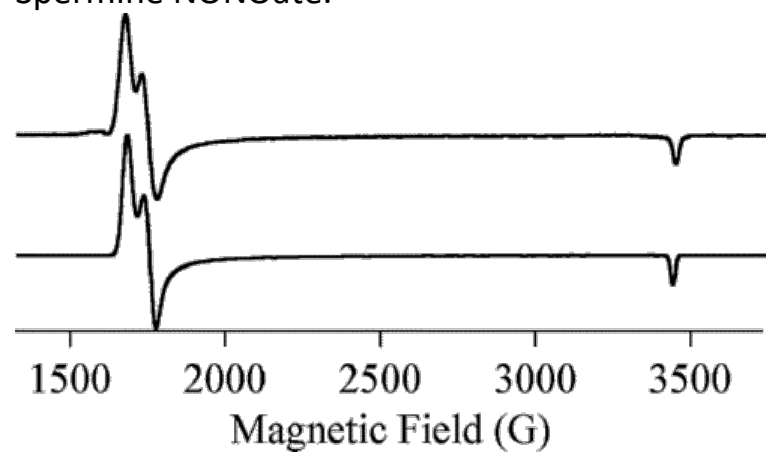

Figure 2 (A) EPR spectrum of the [Fe(PfMetAP-II)]-NO adduct. (B) Simulation of part A assuming an isotropic $g=$ $2.014, E / D=0.0145$. All spectra were recorded at $10 \mathrm{~K}$ using $1 \mathrm{~mW}$ microwave power, a $100 \mathrm{kHz}$ modulation frequency, a $1.26 \mathrm{mT}$ field modulation amplitude, and a $10 \mathrm{mT} \mathrm{s}^{-1}$ sweep rate. 
An important mechanistic question for MetAPs is "Can both substrate and the nucleophilic water/hydroxide bind to the active site divalent metal ion?" In order to answer this question, butane boronic acid (BuBA) was used as a substrate-analogue, ${ }^{11,12}$ and the binding of BuBA to the Fe(II)-NO PfMetAP-II complex was monitored by electronic absorption and EPR spectroscopies. BuBA is a weak competitive inhibitor of [Fe(II)(PfMetAP-II)] with a $K_{\mathrm{i}}$ of $3.5 \mathrm{mM}$. The addition of saturating amounts of BuBA to [Fe(PfMetAP-II)]-NO altered the molar absorptivities of the three observed maxima in the UV-vis spectrum but did not markedly shift any of the maxima. These data suggest that BuBA binds to the Fe(II)-NO center in PfMetAP-II, does not displace NO, and does not change the coordination number.

The EPR spectrum of [Fe(PfMetAP-II)]-NO + BuBA is markedly different from that observed for [Fe(PfMetAPII)]-NO (Figure 3). This new signal exhibits an intense feature at $g=4.2$ with additional features at $g_{\text {eff }}=8.4$ and $g_{\text {eff }}=5.25$. This new species accounts for approximately $80 \%$ of the total signal intensity at $10 \mathrm{~K}$ while the EPR signal from the [Fe(PfMetAP-II)]-NO complex with $g_{\text {eff }}=4.05,3.89$, and 2.0 accounts for $\sim 20 \%$ of the spins in the sample. On the basis of early experimental and theoretical studies, ${ }^{13,14}$ the new features observed in the [Fe(PfMetAP-II)]-NO + BuBA EPR spectrum can be assigned to a single, protein bound high-spin $(S=5 / 2) \mathrm{Fe}(\mathrm{III})$. Compared to the ubiquitous " $g=4.3$ " signal often seen due to adventitious Fe(III), the EPR spectrum of [Fe(PfMetAP-II)]-NO + BuBA is unusual in two respects. First, prominent maxima occur at $g_{\text {eff }}=8.4,5.25$, and 4.2, rather than $9.68,6.0$, and 4.29 , expected for the weak-field limit. ${ }^{15}$ Second, these features are more intense, compared to the $g \sim 4.3$ resonance, than is usually observed for high-spin Fe(III) centers. In fact, both of these observations can be rationalized by considering $D$-strain that is sufficiently high such that a significant population of the molecules no longer satisfy the condition $|D| \gg 6 g H S(=h v)$. Typically, Fe(III) with $|D|$ $\gg$ BgHS exhibits signals at $g \sim 9.7,6.0$, and 4.3 due to extensive $E$-strain. The $g \sim 4.3$ signal is orientation independent $\left(M_{S}=\left| \pm \pm^{3} / 2\right\rangle, E / D=1 / 3\right)$ while the $g \sim 6$ resonance occurs in both $M_{S}=\left| \pm^{3} / 2\right\rangle \quad\left(g_{z}, E / D=0\right)$ and in $M_{s}=| \pm 1 / 2\rangle$ with $x, y$ orientation-independence. The $g \sim 9.7$ resonance occurs in both $M_{s}=| \pm 1 / 2\rangle$ $\left(g_{y}, E / D=1 / 3\right)$ and in $M_{s}=\left| \pm^{5} / 2\right\rangle \quad\left(g_{2}, E / D=1 / 3\right)$. These special conditions result in increased transition probabilities at $g \sim 9.7,6.0$, and 4.3 in the powder spectrum compared to other resonance positions. When $|D|$ becomes comparable to the Zeeman interaction, the resonances at $g_{\text {eff }} \sim 9.7$ and 6.0 are shifted upfield. ${ }^{14}$ Indeed, for the case $|h v / D|=7$, the resonances at $g_{\text {eff }} \sim 9.7,6.0$, and 4.3 are shifted to $8.2,5.3$, and 4.2 , respectively. Further, when the distribution of $D$ is sufficiently wide that species with $|2 D|<\sim 7 \mathrm{~cm}^{-1}$ are present, the transition probabilities in the $\left| \pm^{1} / 2\right\rangle$ and $\left| \pm^{3} / 2\right\rangle$ Kramers' doublets are comparable, and $\left| \pm^{5} / 2\right\rangle$ is also populated, at $10 \mathrm{~K}$. Thus, the intensities of the shifted resonances at $g_{\text {eff }} \sim 8.4$ and 5.25 would be expected to have unusually high intensities compared to the $g_{\text {eff }} \sim 9.7$ and 6.0 resonances for species with $|D| \gg h v$.

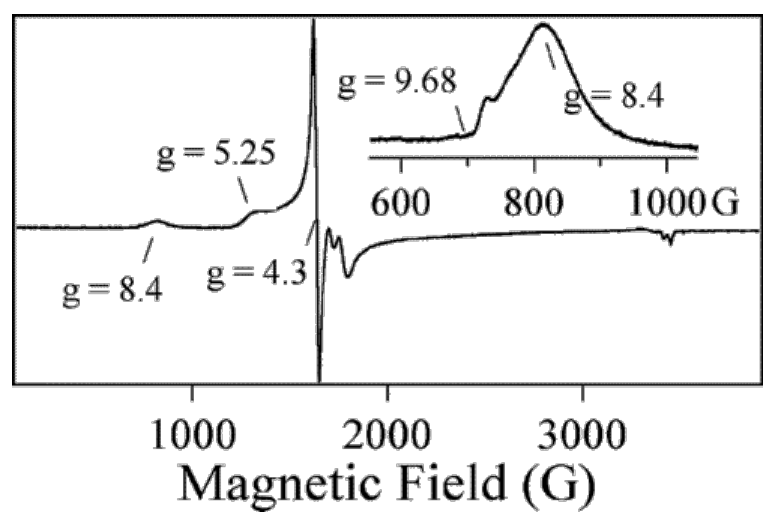

Figure 3 EPR spectra of [Fe(III)(PfMetAP-II)]-NO with BuBA. Inset: Blow-up of the range around $g=6-10$. This spectrum was recorded at $10 \mathrm{~K}$ using $1 \mathrm{~mW}$ microwave power, a $100 \mathrm{kHz}$ modulation frequency, a $1.26 \mathrm{mT}$ field modulation amplitude, and a $10 \mathrm{mT} \mathrm{s}^{-1}$ sweep rate. 
Convincing support for this $E$ - and $D$-strain based explanation of the unusual appearance of the spectrum comes from careful examination of the low-field feature (Figure 3, inset). As $|D|$ approaches $h v, g_{\text {eff }}$ approaches 9.68 (for $E / D=1 / 3$ ), and for all $D>h v, g_{\text {eff }} \sim 9.68 .{ }^{14} A$ sharp cutoff in intensity would therefore be expected as $g_{\text {eff }}$ approaches 9.68 , and no intensity at all should be observed from the $\left| \pm^{1} / 2\right\rangle$ and $\left| \pm^{3} / 2\right\rangle$ Kramers' doublets beyond $g_{\text {eff }}=9.68$. Further, the "pile-up" of resonances as $g_{\text {eff }}$ approaches 9.68 should result in an increase in the intensity of the signal just before the cutoff. This is precisely what is observed for [Fe(PfMetAP)]-NO + BuBA (Figure 3, inset). Moreover, the $S=5 / 2$ signals are largely unchanged as a function of temperature except for some diminution of the $g_{\text {eff }} \sim 9.6$ signal as the temperature increases. This is, in fact, exactly what one would expect for the small value of $D$ that is predicted. On the basis of these data, the observed signal is assigned to a high-spin, mononuclear, $S=5 / 2 \mathrm{Fe}(\mathrm{III})$ species that forms upon binding BuBA to [Fe(PfMetAP--II)]-NO. Since the spin system changes from $S=3 / 2$ to $S=5 / 2$, the Fe(II) center and NO must become uncoupled forming an $\mathrm{Fe}^{3+} S=5 / 2$ center and an NO. Moreover, the observed signal is very unique and markedly different from [Fe(III)(PfMetAP-II)], since it exhibits an unusually low $D$ value, suggesting that NO is still in the coordination sphere of the iron center, but is not covalently bound. These data suggest that $\mathrm{NO}^{-}$forms but must be partially dissociated from the active site metal ion upon the addition of BuBA with the concomitant oxidation of [Fe(PfMetAP-II)] to [Fe(III)(PfMetAP-II)]. The formation and dissociation of NO forming an Fe(III) center is not unprecedented. Orville et al. ${ }^{8}$ showed that the protocatechuate 3,4-dioxygenase NO complex can undergo photodissociation, resulting in the loss of the $S=3 / 2$ EPR signal and the formation of a new signal from an $S=5 / 2$ species. Since the boron atom of BuBA is $\mathrm{sp}^{2}$ hybridized with a trigonal planar geometry, the $\mathrm{p}_{z}$ orbital is empty making the boron atom a good Lewis acid. Furthermore, upon binding BuBA to the Fe(II)-NO center of PfMetAP-II, the boron atom will become even more electron deficient, making it even more susceptible to nucleophilic attack. $\mathrm{NO}$ in $\{\mathrm{FeNO}\}^{7}$ complexes can be described as $\mathrm{NO}^{-}(S=1)$ bound to an Fe(III) center through the $4 \sigma^{+}$molecular orbital of $\mathrm{NO}^{-}$, and the binding of $\mathrm{BuBA}$ to $\left\{\mathrm{FeNO}^{7}\right.$ weakens this binding interaction, thus partially breaking the Fe-NO bond. Since the empty $\mathrm{p}_{z}$ orbital of boron is nearby, it can accept some electron density from NO; thus stabilizing it and making the iron center more ferric in character (Scheme 1).
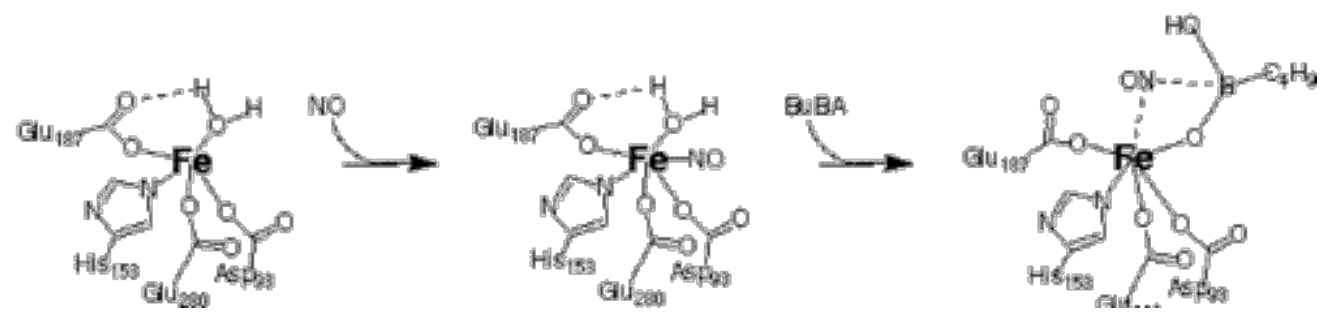

Scheme 1

In conclusion, NO interacts with [Fe(II)(PfMetAP-II)] and directly binds to the $\mathrm{Fe}(\mathrm{II})$ center forming an $\{\mathrm{FeNO}\}^{7}$ complex. The coordination geometry of the $\mathrm{Fe}(\mathrm{II})$ center in [Fe(PfMetAP-II)] expands from five to six upon binding NO, suggesting the retention of a metal-bound water/hydroxide. The addition of BuBA to [Fe(PfMetAP-II)]-NO displaces the coordinated water molecule forming a six-coordinate adduct; however, BuBA appears to undergo nucleophilic attack by NO which mimics an intermediate step between the Michaelis complex and the tetrahedral transition-state. The proposed structure would be similar to the crystallographically characterized BuBA complex of the aminopeptidase from Aeromonasproteolytica (AAP). ${ }^{12}$ Using this structure as a model, the nucleophilic water/hydroxide in [Fe(II)(PfMetAP-II)] is likely metal centered, and both the nucleophile and the substrate reside on the same Fe(II) center in type-II MetAPs. These data are very significant since they provide the first evidence that both nucleophile and substrate can bind to the catalytic metal center in MetAPs. 


\section{Acknowledgment}

This work was supported by the National Institutes of Health (GM-56495, R.C.H.; AI056231, B.B.) and the National Science Foundation (CHE-0240810, R.C.H.). The Bruker ESP-300E EPR spectrometer was purchased with funds provided by the National Science Foundation (BIR-9413530, R.C.H.), and XSophe was purchased with funds from the National Institutes of Health (EB001980, B.B.).

\section{References}

1 Meinnel, T.; Mechulam, Y.; Blanquet, S. Biochimie1993, 75, 1061-1075.

2 Bradshaw, R. A.; Brickey, W. W.; Walker, K. W. TIBS1998, 23, 263-267.

3 Arfin, S. M.; Bradshaw, R. A. Biochemistry1988, 27 (21), 7979-7984.

4 Kruger, E. A.; Figg, W. D. Expert Opin. Invest. Drugs2000, 9, 1383-1395.

5 Cosper, N. J.; D'souza, V.; Scott, R.; Holz, R. C. Biochemistry2001, 40, 13302-13309.

6 Meng, L.; Ruebush, S.; D'souza, V. M.; Copik, A. J.; Tsunasawa, S.; Holz, R. C. Biochemistry2002, 41, 7199-7208. 7 Brown, C. A.; Pavlosky, M. A.; Westre, T. E.; Zhang, Y.; Hedman, B.; Hodgson, K. O.; Solomon, E. I. J. Am. Chem. Soc.1995, 117, 715-732.

8 Orville, A. M.; Lipscomb, J. D. J. Biol. Chem.1993, 268, 8596-8607.

9 Jackson, T. A.; Yikilmaz, E.; Miller, A. F.; Brunold, T. C. J. Am. Chem. Soc. 2003, 125, 8348-8363.

10 Enemark, J. H.; Feltham, R. D. Coord. Chem. Rev.1974, 13, 339-406.

11 Bennett, B.; Holz, R. C. Biochemistry1997, 36, 9837-9846.

12 DePaola, C.; Bennett, B.; Holz, R. C.; Ringe, D.; Petsko, G. Biochemistry1999, 38, 9048-9053.

13 Castner, T. J.; Newell, G. S.; Holton, W. C.; C. P., S. J. Chem. Phys.1966, 32, 668-673.

14 Aasa, R. J. Chem. Phys.1970, 52, 3919-3930.

15 Hagen, W. R. Adv. Inorg. Chem.1982, 38, 165-222. 